\title{
Network analysis: a way forward for understanding COPD multimorbidity
}

\author{
Rosa Faner ${ }^{1,2}$ and Alvar Agustí $i^{1,2,3}$
}

Affiliations: ${ }^{1}$ Fundació Clínic per a la Recerca Biomèdica, Institut d'investigacions Biomèdiques August $\mathrm{Pi}$ i Sunyer (IDIBAPS), Barcelona, Spain. ${ }^{2}$ CIBER Enfermedades Respiratorias (CIBERES), Spain. ${ }^{3}$ Pulmonary Service, Thorax Institute, Hospital Clinic, University of Barcelona, Barcelona, Spain.

Correspondence: Alvar Agustí, Thorax Institute, Hospital Clinic, Villarroel 170 (Escalera 3, Planta 5), Barcelona 08036, Spain. E-mail: alvar.agustiđclinic.ub.es

@ERSpublications

Network analysis of comorbidities in COPD patients provides new insights into the pathobiology of disease http://ow.ly/Ph44T

Patients with chronic obstructive pulmonary disease (COPD) often suffer other concomitant diseases that negatively impact their prognosis and health status [1]. However, it is unclear if they are causally related to COPD or result from shared risk factors, such as ageing, smoking and/or inactivity [2]. Likewise, the precise underlying molecular mechanisms are also unclear [3], hence limiting targeted therapeutic interventions. The use of network analysis can help to elucidate this conundrum [4].

Network analysis allows the creation of molecular and phenotypic networks that could provide new insights into the pathobiology of human diseases [4-6]. Hidalgo et al. [7] used it to investigate comorbidity relationships in the Medicare database, which included more than 30 million patient records, and showed that patients develop diseases close in the phenotypic network to those they already have, and that patients diagnosed with diseases which are more highly connected in the network tend to die sooner than those affected by less connected diseases. These observations clearly support the concept that comorbidities in the general population do not occur by chance and that, most likely, there should be underlying molecular mechanisms that facilitate (or prevent) their occurrence.

GrosDidier et al. [3] also used network analysis to explore the potential molecular mechanisms underlying multimorbidity in COPD. By mining available literature, they found that the 16 most frequent comorbidities of COPD do indeed share many genes, proteins and biological pathways. Furthermore, they also showed that chemicals contained in the tobacco exposome target about $70 \%$ of the identified proteins that participate in these COPD multimorbidities [3]. In this issue of the European Respiratory Journal, Divo et al. [8] expanded their previous work in the field of COPD multimorbidity [9] and also used network analysis to explore the associations between 79 comorbidities and five predefined clinical characteristics (age, body mass index (BMI), severity of breathlessness, level of airflow limitation and exercise capacity) in the BODE cohort (1969 COPD patients) and 316 non-COPD controls of similar age. Results confirmed that the prevalence of comorbidities was higher in COPD patients than in controls but showed that the comorbidity network was more dense (i.e. it had more nodes and edges) and involved more diseases (some of them with unique links) in COPD [8]. Importantly, the COPD comorbidity network was found to have a "scale free" architecture [8]. This indicates the existence of a few, highly connected nodes (diseases) in the network (so-called "hubs") that may play a key pathogenic role by holding the network and contributing to its stability [10]. Based on this observation, Divo et al. [8] propose that these hubs could be targeted for specific correcting interventions. Finally, using network analysis Divo et al. [8] also identified a number of "modules" in the comorbidity network, including a cardiovascular one (observed both in COPD and controls, but more dense and prevalent in the former), a module associated with younger and current smoker COPD patients, a module characterised by mild-moderate airflow limitation and metabolic

Received: April 072015 | Accepted: April 202015

Conflict of interest: Disclosures can be found alongside the online version of this article at erj.ersjournals.com

Copyright $\odot$ ERS 2015 
syndrome with high BMI and, finally, a less dense module centred around gastro-oesophageal reflux. Interestingly, some of these modules reproduce previous findings that used different analytical strategies (cluster analysis) in other cohorts $[11,12]$, thus reinforcing the validity of the observations by Divo et al. [8], who suggested that the diseases forming these modules could be targeted for screening.

In all, therefore, the work by Divo et al. [8] reported in this issue of the European Respiratory Journal, in combination with some other recent publications $[3,7,11,12]$, clearly indicate that comorbidities in COPD do not occur by chance, that different comorbidities cluster with clinical symptoms, and that this is not observed to the same degree in individuals of similar age without COPD. Future research is required to identify the common genetic/environmental basis of multimorbidity in COPD in order to identify novel therapeutic and/or preventive targets.

\section{References}

1 Vestbo J, Hurd SS, Agusti AG, et al. Global Strategy for the Diagnosis, Management and Prevention of Chronic Obstructive Pulmonary Disease. GOLD Executive Summary. Am J Respir Crit Care Med 2013; 187: 347-365.

2 Faner R, Cruz T, López-Giraldo A, et al. Network medicine, multimorbidity and the lung in the elderly. Eur Respir J 2014; 44: 775-788.

3 Grosdidier S, Ferrer A, Faner R, et al. Network medicine analysis of COPD multimorbidities. Respir Res 2014; 15: 111.

4 Diez D, Agusti A, Wheelock CE. Network analysis in the investigation of chronic respiratory diseases: from basics to application. Am J Respir Crit Care Med 2014; 190: 981-988.

5 Barabasi AL, Gulbahce N, Loscalzo J. Network medicine: a network-based approach to human disease. Nat Rev Genet 2011; 12: 56-68.

6 Agusti A, Anto JM, Auffray C, et al. Personalized respiratory medicine: exploring the horizon, addressing the issues. Am J Respir Crit Care Med 2015; 191: 391-401.

7 Hidalgo CA, Blumm N, Barabasi AL, et al. A dynamic network approach for the study of human phenotypes. PLoS Comput Biol 2009; 5: e1000353.

8 Divo MJ, Casanova C, Marin JM, et al. COPD comorbidities network. Eur Respir J 2015; 46: 640-650.

9 Divo M, Cote C, de Torres JP, et al. Comorbidities and risk of mortality in patients with chronic obstructive pulmonary disease. Am J Respir Crit Care Med 2012; 186: 155-161.

10 Agusti A, Sobradillo P, Celli B. Addressing the complexity of chronic obstructive pulmonary disease: from phenotypes and biomarkers to scale-free networks, systems biology, and P4 medicine. Am J Respir Crit Care Med 2011; 183: 1129-1137.

11 Garcia-Aymerich J, Gomez FP, Benet M, et al. Identification and prospective validation of clinically relevant chronic obstructive pulmonary disease (COPD) subtypes. Thorax 2011; 66: 430-437.

12 Rennard SI, Locantore N, Delafont B, et al. Identification of five COPD subgroups with different prognoses in the ECLIPSE cohort using cluster analysis. Ann Am Thorac Soc 2015; 12: 303-312. 